Instituto Internacional de Investigación y Desarrollo Tecnológico Educativo INDTEC, C.A.

DOI: https://doi.org/10.29394/scientific.issn.2542-2987.2017.0.0.18.336-354

OAI-PMH: http://www.indteca.com/ojs/index.php/Revista Scientific/oai

\title{
Estrategias Didácticas para el Fortalecimiento de los Valores Ambientalistas
}

Autora: Marilin del Carmen González Castillo Universidad Pedagógica Experimental Libertador, UPEL mari3@hotmail.com Barinas, Venezuela

\section{Resumen}

El presente estudio tiene como objetivo aplicar estrategias didácticas para el fortalecimiento de los valores ambientalistas en los docentes de la Institución Educativa Casa de los Niños "Linda Barinas", ubicada en la parroquia el Carmen, del municipio Barinas, estado Barinas. La naturaleza del estudio se insertó en el paradigma cualitativo, el tipo de investigación en una investigación acción apoya en un estudio de campo. Los informantes clave fueron cinco (05) docentes que laboran en la institución antes mencionada. La técnica para la recolección de la información a través de una entrevista en profundidad. La técnica y análisis de la información se realizó por medio de la categorización, triangulación y teorización. El estudio permitió concluir: los docentes desconocen del empleó de estrategias de enseñanzas, estrategias de aprendizaje en el abordaje de la Educación Ambiental. Además, la institución carece de la promoción de acciones educativas que contribuyan con el mejoramiento del ambiente. Igualmente, se carece de una actitud positiva hacia el ambiente, en la cual cada integrante de la institución se apropie de los problemas ambientales existente en la escuela. En la ejecución del plan de acción los talleres de formación efectuados a los docentes en cuanto a las estrategias didácticas, valores ambientalistas y educación ambiental, produjeron una motivación e interés de cómo poner en práctica, ese conocimiento, al momento de planificar, organizar y controlar.

Palabras clave: estrategias didácticas; fortalecimiento; valores ambientalistas. 


\title{
Teaching Strategies for Strengthening Environmental Values
}

\begin{abstract}
This study aims to apply teaching strategies for strengthening the environmental values in the teachers of School Children's House "Linda Barinas" located in the Carmen parish, municipality Barinas, Barinas state. The nature of the study was inserted into the qualitative paradigm, the kind of research in an action research based on a field study. Key informants were five (05) teachers working in the aforementioned institution. The technique for collecting information through an in-depth interview. The technique and data analysis was performed by means of categorization, triangulation and theorizing. The study led to the conclusion: teachers are unaware of employed teaching strategies, learning strategies in addressing environmental education. In addition, the institution lacks the promotion of educational activities that contribute to improving the environment. Similarly, the absence of a positive attitude towards the environment in which every member of the institution appropriates the existing environmental problems at school. In implementing the action plan training workshops conducted for teachers regarding teaching strategies, environmental values and environmental education, produced a motivation and interest of how to implement that knowledge, when planning, organizing and controlling.
\end{abstract}

Keywords: teaching strategies; strengthening; environmental values. 


\section{Introducción}

La educación ambiental es un campo en continua construcción, su génesis se remonta al viaje inaugural de la hominización transcurriendo por todas las épocas y, adquiriendo estatus con la percepción de la crisis ambiental contemporánea. Durante el último siglo, el escenario ambiental irrumpe en los espacios políticos, científicos y educativos, con una trayectoria signada por influencias conceptuales, ocupando cada vez mayores espacios de reflexión y actuación en la comprensión de los cambios globales de estos tiempos, así como para la preparación de mentalidades que le confieran al ser habilidades para la resolución de las dificultades ambientales, abriendo de esta manera el camino hacia un futuro sustentable, equitativo y democrático.

Las primeras manifestaciones referidas a la educación ambiental estaban atadas a las concepciones dominantes, diseñadas en el mundo subdesarrollado, particularmente conservacionistas, sometido al poder del mercado, a la racionalidad ante la que se retrae el pensamiento, se disuelve el sentido y se paraliza la acción; a un poder concentrador de riquezas, generador de insustentabilidad y desigualdad. La educación ambiental reconoce su origen en la forma orientadora de la razón, encontrándose con un pensamiento megaordenador ejercido a través de una matriz disciplinaria fundada en la lógica positivista $y$, convertida en ideal pedagógico para incorporarse en los escenarios educativos, sociales y políticos.

Por otra parte, el hombre ha ido alcanzado niveles superiores de diferenciación con respecto a otros organismos vivos, fundamentalmente, por la conciencia reflexiva que le acompaña la cual to ha conducido a instalarse con propiedad sobre los ecosistemas a través de todo un andamiaje adaptativo y, aún más allá, de transformación del medio natural del que se vale. En este interactuar la ciencia ha dado especial importancia a todo el quehacer humano, matizándolo con atributos como un ser eminentemente cultural. 
Aún, en la actualidad, luego de reformas insustentables, sigue expresándose la fragmentación en la educación ambiental, reflejo del pensamiento de la ciencia clásica edificada en la modernidad, la cual ha mostrado su incontenible proceso entrópico, ocasionando efectos de grandes magnitudes como lo son: la desvaloración material y espiritual en los grupos humanos, especialmente, los que habitan en los centros urbanos, el aumento de la violencia, la delincuencia y el detrimento en la calidad de vida, por citar algunos.

En este orden de ideas, Venezuela no está ausente de estas responsabilidades, es por ello que en el aspecto educativo en los últimos diez (10) años se han experimentado profundos cambios en materia de Educación Ambiental y la manera de cómo abordar los problemas del desequilibrio de la naturaleza. Esto se traduce que el proceso de educación ambiental debe desarrollarse a través de valores ambientalistas, que el conocimiento tenga significado, al respecto, Núñez (2014), plantea:

Darle cabida a la cultura ambiental en el sistema educativo venezolano es enfrentar nuevos cambios paradigmáticos hacia un verdadero proyecto pedagógico, reflexivo y complejo, dinámico y en construcción permanente, dirigido al ser humano como persona y como parte fundamental de la comunidad, a fin de que este pueda aprender los conocimientos conceptuales, actitudinales, procedimentales, preceptúales e interactivo-convivenciales, con la finalidad de emprender acciones que conduzcan a una conciencia ambientalmente responsable y contribuya con la calidad de vida y al desarrollo sostenible de la nación y del planeta (pág. 89).

Es decir, el autor expresa que hay que enrumbar el proceso educativo, acezar a él como fuente de promoción de valores, principios, creencias, en el cual se le una visión regeneradora al país, a las organizaciones, familias y al ser como tal. Por lo cual, la articulación de los preceptos de los valores ambientales a los principios de la educación ambiental genera un nuevo 
significado, en el que se evidencia a la educación, pero no sólo a la educación formal, sino también, a la educación de la calle, la familia, el trabajo, la iglesia, el deporte, entre otros, como instrumentos del aparato social que determina la común unidad de la vida.

Por consiguiente, las estrategias didácticas, según Montilla (2015) expone que las estrategias didácticas "es el conjunto de procedimientos, apoyados en técnicas de enseñanza que tienen por objeto llevar a un buen término la acción didáctica, es decir; alcanzar los objetivos de aprendizaje" (pág. 71). Es decir, se hace referencia a una planificación en el proceso de enseñanza y aprendizaje, lo cual implica un conjunto de decisiones que el docente debe tomar de manera consciente y reflexiva, con relación a las técnicas y actividades que puede utilizar para llegar a las metas de su curso.

De allí que, el objetivo real de las estrategias didácticas es generar cambios positivos, en el proceso de enseñanza-aprendizaje en los estudiantes, con la finalidad de evitar prácticas pedagógicas rutinarias que afecten a la educación venezolana y los cambios deben iniciarse de inmediato en las acciones que realicen los docentes en sus aulas. Solo así se podrá fortalecer la calidad de los aprendizajes, a base de las diferentes estrategias didácticas que constituyen un paso hacia delante en el mejoramiento de la formación del educando.

En consecuencia, el docente es un profesional que debe poseer dominio de un saber especifico y complejo (el pedagógico), que comprende los procesos en que está inserto, que decide con niveles de autonomía sobre contenidos, métodos y técnicas, de acuerdo a la heterogeneidad de los estudiantes; organizando contextos de aprendizaje, interviniendo de distintas maneras para favorecer procesos de construcción de conocimientos desde las necesidades particulares de cada uno de sus estudiantes.

Evidentemente, la misión del docente es contribuir al crecimiento de sus estudiantes. Asistir, desde los espacios estructurados para la enseñanza 
sistemática, al desarrollo integral de las personas. Su función es mediar y asistir en el proceso por el cual lo estudiantes desarrollan sus conocimientos, sus capacidades, sus destrezas, actitudes y valores, en el marco de un comportamiento que valora a otros y respeta los derechos individuales y sociales.

Por lo tanto, las estrategias didácticas en el fortalecimiento de los valores ambientalistas invita al educador a visionar la construcción teórica desde la concepción ambiental hasta sus valores; invita atreverse a observar la relación ser-ambiente-sociedad desde una perspectiva pluralista, de adentro hacia afuera y viceversa, a través de la correlación de ideas antagónicas, con una amplitud en la perspectiva y comprensión del pensamiento y comportamiento humano asociado al funcionamiento multidimensional del ambiente: físico, social, cultural, económico, político y espiritual, constituyendo el cimiento en la edificación de un nuevo discurso ambiental.

Lo antes expuesto no ocurre en las instituciones del estado Barinas, razón por la cual Odremán (2015) expone que "en las instituciones educativas que hacen vida en el municipio Barinas, específicamente, en la Escuelas que pertenecen al Eje Josefa Camejo, ameritan de la aplicación de acciones pedagógicas en el fortalecimiento de los valores ambientalistas" (pág. 45). En otras, palabras los gerentes ni docentes se ocupan por mejorar e incorporar estrategias en función de mejorar el ambiente.

De allí que, los docentes que laboran en la Institución Educativa Casa de los Niños "Linda Barinas", ubicada en la parroquia el Carmen, del municipio Barinas, a través de la observación directa se percibe, aun cuando cuenta con un personal calificado, requiere de una mayor participación en la Educación Ambiental, a través de estrategias didácticas que le permitan al niño y a la niña, de acuerdo a su interés y necesidad, el desarrollo de un aprendizaje significativo, propiciando valores fundamentales como: el amor, el respeto, la cooperación y la solidaridad hacia el ambiente. 
Por otra parte, en entrevistas no formales realizadas a los docentes indicaron que carecen de estrategias didácticas que contribuyan con los valores ambientales. Además, expusieron que el ambiente de esta institución se encuentra bastante deteriorado, tanto dentro como en los alrededores, puesto que aún se inunda cuando llueve por la falta de buenos drenajes. Otra problemática, es la quema de basura por parte de los vecinos cercanos a la institución donde en algunas ocasiones la misma se ha visto afectada ya que las Ilamas han llegado a la parte trasera d la misma quemando algunos árboles de teca.

Por lo antes expuesto surge la inquietud de realizar un trabajo de investigación que tiene como objetivo aplicar estrategias didácticas para el fortalecimiento de los valores ambientalistas en los docentes de la Institución Educativa Casa de los Niños "Linda Barinas", ubicada en la parroquia el Carmen, del municipio Barinas, estado Barinas. Por consiguiente, se plantean las siguientes interrogantes de investigación:

¿Cómo será la aplicación de estrategias didácticas para el fortalecimiento de los valores ambientalistas en los estudiantes de Educación Primaria, por parte de los docentes del Institución Educativa Casa de los Niños "Linda Barinas"?, ¿Cómo será la planificación de las estrategias didácticas para el fortalecimiento de los valores ambientalistas en los estudiantes de Educación Primaria?, ¿Qué mecanismos se emplearan en la ejecución de las estrategias didácticas para el fortalecimiento de los valores ambientalistas en los estudiantes de Educación Primaria?¿Cómo será la evaluación de la ejecución de las estrategias didácticas para el fortalecimiento de los valores ambientalistas en los estudiantes de Educación Primaria. 


\subsection{Objetivos de la Investigación}

\subsubsection{Objetivo General}

Aplicar estrategias didácticas para el fortalecimiento de los valores ambientalistas en los docentes de la Institución Educativa Casa de los Niños "Linda Barinas", ubicada en la parroquia el Carmen, del municipio Barinas, estado Barinas.

\section{Referentes Teóricos}

\subsection{Estrategias Didácticas}

La palabra estrategia ocasionalmente se ha venido aplicando en el sentido más amplio en diversos campos y procedimientos, sobre todo para explicar la forma de conducir un proceso sea política, económica, militar, educativo entre otros. Ferreiro (2012) su origen procede del "ámbito militar y significa literalmente el arte de dirigir las operaciones militares" (pág. 59).

Para este autor con el desarrollo del paradigma cognitivo así como el constructivismo el concepto de estrategia ha sido transferido creativamente al ámbito educativo en el marco de la propuesta de enseñar y aprender a pensar, en ese sentido el autor antes mencionado le da especial importancia como componente esencial del proceso de enseñanza y aprendizaje, Ferreiro (ob.cit): sistema de actividades (acciones y operaciones) que permite - con economía de esfuerzo y recursos-la realización de una tarea con la calidad requerida dada la flexibilidad y adaptabilidad que ellas ofrecen en su empleo a las condiciones existentes (pág. 60).

De lo anterior expuesto puede decirse que en términos más amplio las estrategias son el orden en el cumplimiento del trabajo, permitiendo su buena ejecución y ahorro de tiempo y errores, es decir, que la buena selección de una estrategia permite economizar tiempo, recurso y esfuerzo y aun mas, la seguridad de conseguir lo que se quiere y por la vía más adecuada posible. 
Siguiendo con ese mismo orden de ideas Rodríguez (2012), señala que teóricamente las estrategias se definen desde tres puntos de vista (a) militar, (b) empresarial y (c) educacional, en función de dos enfoques: el enfoque clásico o de las definiciones que solo incluyen a los medios y (b) el moderno que incluye, los medios, los objetivos y los demás componentes en un plan o programa. (pág. 133). El autor refiere que las estrategias pueden visualizarse desde tres ópticas: militar, empresarial y en el ámbito educativo.

Sin embargo, para efectos de esta investigación es importante definir las estrategias desde el punto de vista educacional moderno que pertenece a los postulados de Kindsvatter y Wilen e Ishler (1988), citado por Rodríguez (2012), quien al mismo tiempo la define como el procedimiento para desarrollar la instrucción permitiendo al docente tomar decisiones respecto a su rol, al del estudiante, seleccionar métodos y materiales; es decir es el arsenal pedagógico que permite al docente organizar los objetivos de la instrucción, los métodos, las técnicas y la evaluación de los aprendizajes.

De lo antes expuesto, se deduce que desde este enfoque educacional, las estrategias se convierten en un componente esencial del proceso de enseñanza y aprendizaje, por lo cual existen diversidad de estrategias, pero en el plano educativo Ferreiro (2012) le da mayor relevancia a las estrategias de enseñanza 0 instruccionales y las estrategias de aprendizajes, quien establece una clara definición de ambas. Con respecto a las estrategias de enseñanza este autor las denominas instruccionales o didácticas a fin de definir el procedimiento empleado por el maestro para hacer posible el aprendizaje de sus alumnos. Por otra parte, las estrategias de aprendizajes, constituyen los procedimientos eminentemente mentales que el alumno sigue para aprender, procesar la información y aprovecharla significativamente.

Como complemento a esta definición de estrategias didácticas, Ferrándiz (2012), establece una definición clara de las estrategias didácticas cuando señala que son "el conjunto organizado de procedimientos, técnicas, 
recursos y actividades que tienen como propósito contribuir al desarrollo conceptual de los alumnos, orientadas al predominio de aprendizajes significativos" (pág. 48). La autora señala que las estrategias didácticas toman en cuenta las características de los alumnos, los objetivos propuestos en las asignaturas y la flexibilidad del currículo.

Por lo tanto, es posible entonces definir a las estrategias didácticas como la unión de acciones bien intencionadas, ordenadas, secuenciadas y sistematizadas de actividades y recursos expresados a través de métodos y técnicas del cual se apoya el docente en su práctica educativa diaria para facilitar aprendizajes significativos. Es importante acotar que las estrategias permiten la organización de los contenidos a desarrollar por el docente, de ahí que es necesario contar con estrategias adecuadas para iniciar la clase, desarrollar el tema y por supuesto cerrar o concluir la lección, como los tres grandes momentos de la clase y que Ferrándiz (ob.cit) los define como los componentes de una estrategia didáctica.

\subsection{Modalidades de las Estrategias Didácticas}

La enseñanza pero sin duda, si no se acota los espacios de acción y se interesan en modelos educativos más amplios que tomen en cuenta la realidad y la plausibilidad psicológica a la vez que la validez de los contextos reales, las estrategias didácticas no estaría solamente referidas a las labores de planeamiento docentes sino que se vincularía con todo el quehacer educativo y sin duda a modo de encaje sistémico en la que debe relacionarse de manera directa con las estrategias de aprendizaje de los estudiantes.

Para Bustos (2013) como es bien sabido por los docentes, "en toda planificación educativa se toman en cuenta las características e intereses de los alumnos, las condiciones y oportunidades que existan en los espacios educativos y los recursos con los cuáles se cuenta" (pág. 43). Partiendo de allí, esta estrategia didáctica admite la adecuación de la misma a esas 
particularidades, pero sin obviar la vinculación de la docencia con la investigación y la extensión. Afirma esta misma autora que consecuencialmente en el contacto inicial con los estudiantes se introduce el contenido de la asignatura y los escenarios circunstanciales que condicionan el desarrollo del semestre.

\subsection{Valores Ambientalistas}

Son muchos los autores que han escrito sobre los valores, cada uno de ellos le ha dado un enfoque diferente, sin embargo coinciden en que todos los seres humanos no pueden llevar una vida digna si carecen de ellos, por ser parte importante dentro de su formación. Se considera "valor" a aquellas cualidades o características de los objetos, de las acciones o de las instituciones atribuidas y preferidas, seleccionadas o elegidas de manera libre, consciente, que sirven al individuo para orientar sus comportamientos y acciones en la satisfacción de determinadas necesidades.

De allí que, Carreras (2012), los valores sirven para guiar las conductas de las personas, son el fundamento por el cual hacemos o dejamos de hacer una cosa en un determinado momento. Dicho de otra manera, los valores son creencias prescriptivas 0 , si se prefiere principios normativos y duraderos que nos sugieren determinada conducta o un estado final de existencia personal y socialmente preferible a otros.

En este orden de ideas, los valores ambientalistas debe ser motora de la reproducción social y liberadora o potenciadora del crecimiento individual y colectivo que produzca cambios sociales, culturales y políticos, de tal forma que generen el progreso en ámbitos relacionados con la libertad, los derechos, la participación y el control del poder que tengan los ciudadanos. Estas consideraciones son tareas nada simples que se debe plantear desde el ámbito educativo para generar cambios a mediano y largo plazo. 
En la actualidad, en la práctica docente, se enfatiza el desarrollo de objetivos conceptuales y procedimentales y no tanto el fomento de valores actitudinales hacia el ambiente; en realidad debería privilegiarse la búsqueda colectiva de las causas y los efectos de los problemas ambientales para fomentar cambios de actitudes respecto a los problemas que aquejan el entorno de la vida escolar y que, de una manera u otra, afectan negativamente la calidad de la vida.

Por su parte, Nuévalos (2013), define los valores ambientalistas "éstos son inherentes a la necesidad de propiciar las actitudes, valores y conductas necesarias para que, individual o colectivamente, las personas participen de forma eficaz, responsable y solidaria en la prevención y solución de los problemas ambientales" (pág. 56). En otras palabras, los valores ambientalistas buscan favorecer los valores, cualidades que el individuo desarrolla en la prevención del ambiente.

\section{Metodología}

\subsection{Naturaleza de la Investigación}

De acuerdo a las características del problema planteado y objetivos de la investigación, responde metodológicamente al paradigma de tipo cualitativo, apoyado en un a investigación de campo, enfocado en el método de Investigación Acción, porque interpreta descriptivamente la realidad dentro del contexto estudia las categorías de forma simultánea en un momento dado, a tal efecto se pretende aplicar estrategias didácticas para el fortalecimiento de los valores ambientalistas en los docentes de la Institución Educativa Casa de los Niños "Linda Barinas", ubicada en la parroquia el Carmen, del municipio Barinas, estado Barinas. 


\subsection{Tipo y Diseño de la Investigación}

El tipo de investigación que se realizó está enmarcada en una investigación acción. La Universidad Pedagógica Experimental Libertador (2006) define la investigación acción como la producción de conocimientos para guiar la práctica que conlleva a las modificaciones de una realidad dada en el proceso investigativo.

En consecuencia, la acción es parte integral de la investigación, debido a que la información obtenida de la realidad que vive los docentes de la Institución Educativa Casa de los Niños "Linda Barinas", ubicada en la parroquia el Carmen, del municipio Barinas, estado Barinas, fue el punto de partida para la contextualización de estrategias oportunas e innovadoras para solventar la situación problemática que implica la participación conjunta de las personas que van a ser beneficiarias de la investigación y de aquellos quienes van a hacer el diseño, la recolección y la interpretación de los datos para encontrar soluciones a las necesidades y requerimientos.

\subsubsection{Fases de la investigación}

Para abordar las fases de la investigación fue necesario el acercamiento y conocimiento por parte del investigador al espacio geográfico a intervenir, momento en el cual se utilizaron estrategias de comunicación y cooperación para conocer a la colectividad, no se trata de identificar problemas teóricos, ni prácticos a ligera, es socializar en la búsqueda de la pertenencia comunitaria. El proceso de socialización se llevará a cabo en los docentes de la Institución Educativa Casa de los Niños "Linda Barinas", con los informantes clave que por sí solos mostraron sus poténciales de conocimiento dentro de la escuela. Al mismo tiempo se insertaron progresivamente las ideas planteadas por la investigadora a través de temática y el deseo de participación. 


\subsection{Informantes Clave}

Para el desarrollo de las estrategias didácticas para el fortalecimiento de los valores ambientalistas, se fijaron criterios considerando el fenómeno de la investigación y circunstancias situacionales. Al respecto, Pérez (2009) plantea que todos los escenarios y personas son dignos de estudio" (pág. 48). Se consideran cinco (05) informantes clave, conformados por docentes que laboran en la Institución Educativa Casa de los Niños "Linda Barinas", ubicada en la parroquia el Carmen, del municipio Barinas, estado Barinas.

\subsection{Técnicas e instrumentos de Recolección de Información}

La técnica utilizada de acuerdo con la línea de investigación cualitativa fue la observación y la entrevista en profundidad. La observación participativa. Martínez (2009), indica que es la base fundamental para el investigador debido a que le permite indagar y participar con grupos de personas sobre sus experiencias y manera de vivir. La entrevista en profundidad juega un papel importante en este estudio, según Carrasquilla (2012) considera la entrevista como "aquella situación que permite el traspaso de información entre dos o más personas" (pág. 74). Un instrumento técnico adaptando a un diálogo con preguntas abiertas, las cuales deben ser precisas de manera que el entrevistado pueda abrirse a las ideas guardadas en su subconsciente y que resultan valiosas para la investigación.

\subsection{Análisis de los Datos}

El análisis de la información se procesó empleando una matriz de doble entrada en la cual se reflejan cada una de las respuestas aportadas por los informantes clave, a saber cinco docentes que laboran en la Institución Educativa Casa de los Niños "Linda Barinas", ubicada en la parroquia el Carmen, del municipio Barinas, estado Barinas, seleccionados de acuerdo a la experiencia en la labor educativa. 
Cuadro 1. Matriz de Análisis del Contenido de la Información.

\section{Unidad de Análisis: Estrategias Didácticas}

Categoría: Procesamiento Sub-Categoría: Conocimiento de Técnicas. Barinas.

\begin{tabular}{|c|c|c|c|c|c|}
\hline Pregunta & Informante 1 & Informante 2 & Informante 3 & Informante 4 & Informante 5 \\
\hline $\begin{array}{c}\text { 1.- Con base a } \\
\text { su experiencia, } \\
\text { ¿cómo } \\
\text { considera el } \\
\text { conocimiento } \\
\text { de técnicas en } \\
\text { la aplicación de } \\
\text { estrategias } \\
\text { didácticas en el } \\
\text { fortalecimiento } \\
\text { de los valores } \\
\text { ambientales? }\end{array}$ & $\begin{array}{l}\text { El conocimiento } \\
\text { es poco porque } \\
\text { se carece de } \\
\text { técnicas que } \\
\text { contribuyan con } \\
\text { el ambiente. }\end{array}$ & $\begin{array}{l}\text { Desconocimient } \\
\text { o de técnicas en } \\
\text { la aplicación de } \\
\text { estrategias } \\
\text { didácticas en } \\
\text { los valores } \\
\text { ambientales. }\end{array}$ & $\begin{array}{l}\text { No manejo } \\
\text { conocimiento } \\
\text { relacionado con la } \\
\text { aplicación de } \\
\text { estrategias } \\
\text { didácticas... }\end{array}$ & $\begin{array}{c}\text { Mira } \\
\text { conocimiento } \\
\text { relacionado con } \\
\text { eso muy } \\
\text { escaso, no ser } \\
\text { toma en cuenta } \\
\text { los valores } \\
\text { ambientales. }\end{array}$ & $\begin{array}{l}\text { Desconozco de } \\
\text { técnicas para la } \\
\text { aplicación de } \\
\text { valores } \\
\text { ambientales. }\end{array}$ \\
\hline
\end{tabular}

Fuente: El Autor (2016)

\subsubsection{Análisis}

Se evidencia en la pregunta 1 , tres docentes opinan que tienen poco conocimiento de técnicas en la aplicación de estrategias didácticas en el fortalecimiento de valores ambientalistas y sólo dos consideran que tienen desconocimiento sobre la aplicación de la misma. En lo que respecta a la pregunta 2, tres entrevistados respondieron que es importante la aplicación de recursos en el fortalecimiento de los valores ambientalistas y sólo dos educadores señalaron que se carece de la aplicación de recursos. Se infiere 
que existe la necesidad de aplicar técnicas y recursos que contribuyan con el fortalecimiento de los valores ambientalistas por parte de los docentes.

En este sentido, Ferreiro (2012) expone que la aplicación de técnicas y recursos son importantes porque representan herramientas valiosas para el mejoramiento de conducta en un individuo, es decir, genera cambios y transformaciones que pueden ir de beneficios a una colectividad y por ende optimizar la conservación y mantenimiento del ambiente.

\section{Resultados}

Los resultados obtenidos se centraron en que existe la necesidad de aplicar técnicas y recursos que contribuyan con el fortalecimiento de los valores ambientalistas por parte de los docentes. Igualmente, la ausencia de la utilización de estrategias de enseñanza para el fortalecimiento de los valores ambientalistas; así como la necesidad de utilizar estrategias de aprendizajes en la formación de los valores. Así como tampoco incorporan en la planificación diaria la ejecución de estrategias didácticas para formar en valores ambientalista y sólo dos docentes emplean estrategias didáctica como los mapas de conceptos y las discusiones. Se carece de la ejecución, en su planificación diaria, la formación de valores ambientalistas.

\section{Conclusiones}

La entrevista aplicada a los informantes clave reveló la generación de estrategias didáctica para el fortalecimiento de los valores ambientalistas, la misma busca un nuevo enfoque constructivista que le permita a estudiantes y

docentes ser promotores sociales y cohesione entre los integrantes de la comunidad educativa en el mejoramiento del ambiente, a través de estrategias significativas de aprendizaje que contribuyan a consolidar el trabajo comunitario dentro y fuera de la escuela. 
Por lo tanto, se hace necesario aprender a oír, dialogar, participar, trabajar interdisciplinariamente, comunicarse dentro de distintos modos de percepción del contexto escolar y, acercarse a los verdaderos intereses y motivaciones, tanto del contexto escolar como el comunitario. Pero por encima de todo, aprender a respetar el derecho de las personas a construir su propio destino en función a la aplicación de estrategias didácticas que vayan en beneficio de los estudiantes, docentes y demás miembros de la institución.

La ejecución se efectuó con las actividades, por objetivos específicos, indicados en el plan de acción. El ella se ejecutó cada una las estrategias didácticas planificadas, en la cual se usaron dinámicas de grupos para la interacción de los participantes, dinámicas para conformar equipos de trabajo, se entregó material relacionado con las estrategias didácticas, valores ambientalistas y educación ambiental. También, la conformación de la brigada ambientalista, el diálogo de saberes y la implementación de la galería ambientalista fue una sensación entre los docentes y directivos.

Por lo tanto, la participación, interés y motivación de los educadores fue bastante satisfactoria en el aprendizaje de los valores ambientalistas; quedando establecido, en acta, el compromiso de seguir asumiendo la utilización de las estrategias didácticas en la planificación diaria de los docentes. Se allí que, los talleres de formación realizada a los docentes en cuanto a las estrategias didácticas, valores ambientalistas y educación ambiental, produjeron una motivación e interés de cómo poner en práctica, ese conocimiento, al momento de planificar, organizar y controlar, el diseño, elaboración y ejecución de planes en beneficio de los estudiantes.

Es importante destacar que todos los objetivos previstos fueron logrados, dando cumplimiento con todas las actividades planificada, en el marco de desarrollo del plan de acción. Durante la ejecución de las actividades se pudo lograr la participación y la organización de los docentes, se señala que los participantes manifestaron que los talleres llenaron sus expectativas y 
que son de gran utilidad, ya que, actualmente necesitan del fortalecimiento de los valores ambientalistas en los estudiantes.

\section{Referencias}

Bustos, C. (2013). Estrategias Didácticas para la Vinculación Docencia, Investigación y Extensión en la Praxis Educativa. (1era. ed.). Caracas: Colección de textos universitarios LUZ.

Carreras, N. (2012). Los Valores. España: Espasa.

Ferrándiz, M. (2012). Informe de Investigaciones Educativas Volumen XIII. № 1 Año 2009. Caracas: UNA.

Ferreiro, E. (2012). Estrategias Didácticas. Buenos Aires: Fondo de Cultura Económica, S.A.

Martínez, M. (2009). La Investigación Cualitativa Etnográfica en Educación. (3ra ed.). México: Trillas.

Montilla, O. (2015). Estrategias Didácticas. México: Trillas.

Nuévalos, Ñ. (2013). Valores Ambientalistas. Madrid: Humanista.

Núñez, C. (2014). Estado del arte de la educación ambiental en Venezuela. CONHISREMI, Revista Universitaria de Investigación y Diálogo Académico, Volumen 12, Número 2-3.

Odremán, C. (2015). Acciones Pedagógicas en el Fortalecimiento de los Valores Ambientalistas en las instituciones pertenecientes al Eje Josefa Camejo del municipio Barinas. Trabajo de Grado de Maestría, Universidad Fermín Toro. Cabudare, Barquisimeto.

Pérez, G. (2012). Investigación cualitativa. Retos e interrogantes. Madrid: La Muralla.

Rodríguez, T. (2012). Estrategias Didácticas. (4ta. ed.). Buenos Aires: Limusa. 


\section{Marilin del Carmen González Castillo}

e-mail: mari3@hotmail.com

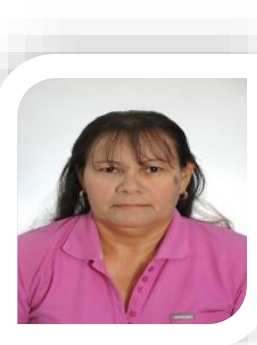

Nacida en Venezuela, residenciada en Barinas, estado Barinas. Estudios realizados: Primaria: Escuela Básica "Don Lino Jiménez", Secundaria: Liceo Bolivariano "Manuel Palacio Fajardo" Diversificado: Liceo Nocturno "Alberto Arvelo Torrealba" (Bachiller en Humanidades) y Superior en la Universidad Pedagógica Experimental Libertador-Instituto de Mejoramiento Profesional del Magisterio, Núcleo Académico Barinas con el Título de Profesor Especialidad en Educación Integral. Algunos cursos realizados: Foro: Ley para la Protección de Niños, Niñas y Adolescentes en Salas de Uso de Internet y otros Multimedia, Taller de Estimulación a Temprana Edad, Jornadas Educación "Aprendiendo a Vivir como Niño o Niña", Taller Nuevos Paradigmas en las Relaciones Humanas, Taller de Linux Básico, Curso de Cuarto Nivel El Hombre y su Ambiente, Congreso Internacional de Investigación e Innovación en Currículo y Formación Docente (como ponente).

El contenido de este manuscrito se difunde bajo una Licencia de Creative Commons ReconocimientoNoComercial-Compartirlgual 4.0 Internacional 